\title{
Metastasis of breast cancer prior to invasion
}

\author{
Tibor $\operatorname{Tot}^{1}{ }^{1}$
}

Received: 29 January 2018 / Accepted: 15 February 2018 / Published online: 19 February 2018

o) Springer Science+Business Media, LLC, part of Springer Nature 2018

To the Editor,

I read with interest the review article of Narod and Sopik in a recent issue of Breast Cancer Research and Treatment [1] discussing the question whether invasion is a necessary step for metastases in breast cancer. I was impressed by their detailed argumentation and brave conclusions. The alternative (parallel) model of breast cancer metastasis they proposed is, however, not a new one. It has been described in a book published by Springer 2011 [2] on the following way: "Mutant stem cells and committed progenitor cells share many characteristics with malignant stem cells. These mobile cells may, in some cases, enter the circulation and be transported to lymph nodes and other organs. Malignant transformation of the cell progeny may require several years or decades, like their counterparts within the breast. Transformation of these relocated mutant progenitor cells prior to the transformation of the intramammary compartment of these cells may give rise to a metastasis of "unknown origin". This concept may represent a possible explanation for, at least some cases of so-called CUP (cancer with unknown primary) syndrome. Such a transformation may also take place decades later than the intramammary transformation." With this short notice, I would like to call your attention to this fact and inform the authors and the readers that identical thoughts to those expressed by Narod and Sopik have been published years ago. I also hope, this may strengthen the idea of possible dissemination of progenitor cells committed to become malignant prior to development of the primary invasive cancer.

\section{References}

1. Narod S, Sopik V (2018) Is invasion a necessary step for metastases in breast cancer? Breast Cancer Res Treat. https://doi. org/10.1007/s10549-017-4644-3

2. Tot T (2011) The theory of the sick lobe. In: Tot T (ed) Breast cancer: a lobar disease. Springer, London

This comment refers to the article available at https://doi. org/10.1007/s10549-017-4644-3.

Tibor Tot

tibor.tot@1tdalarna.se

1 Pathology \& Cytology Dalarna, Uppsala University, Falun, Sweden 